\title{
Low-frequency quadrupole impedance of undulators and wigglers
}

\author{
A. Blednykh, G. Bassi, Y. Hidaka, and V. Smaluk \\ Brookhaven National Laboratory, Upton, New York 11973, USA
}

G. Stupakov

SLAC National Accelerator Laboratory, Menlo Park, California 94025, USA

(Received 27 May 2016; published 25 October 2016)

\begin{abstract}
An analytical expression of the low-frequency quadrupole impedance for undulators and wigglers is derived and benchmarked against beam-based impedance measurements done at the $3 \mathrm{GeV}$ NSLS-II storage ring. The adopted theoretical model, valid for an arbitrary number of electromagnetic layers with parallel geometry, allows to calculate the quadrupole impedance for arbitrary values of the magnetic permeability $\mu_{r}$. In the comparison of the analytical results with the measurements for variable magnet gaps, two limit cases of the permeability have been studied: the case of perfect magnets $\left(\mu_{r} \rightarrow \infty\right)$, and the case in which the magnets are fully saturated $\left(\mu_{r}=1\right)$.
\end{abstract}

DOI: 10.1103/PhysRevAccelBeams.19.104401

\section{INTRODUCTION}

For efficient and high-performance operations of particle accelerators, one of the crucial requirements is particle beam stability $[1,2]$. Beam instabilities can be the result of a particle beam interaction with vacuum chamber components. The basic mechanism of the instability can be described as follows. A beam passing through a vacuum chamber generates electromagnetic fields called the wakefields. If the vacuum chamber is nonuniform or has finite resistivity, the wakefields can act back on the beam itself causing beam instabilities. In frequency domain, the beamwake interaction is described using the concept of coupling impedance [3], where a vacuum chamber component is represented by longitudinal and transverse impedances. In the analysis of the vacuum chamber impedance, one can separate contributions due to changes in vacuum enclosure (geometric impedance) and the finite surface resistivity (resistive-wall impedance). Analysis of the beam-impedance interaction usually can be done separately for the broadband impedance (short-range wake) and narrow-band impedance (long-range wake). The broadband impedance causes single-bunch effects, whereas the narrow-band one causes multibunch effects.

For a vacuum chamber with mirror symmetry relative to the horizontal $(y=0)$ and vertical $(x=0)$ planes, the transverse impedance $Z_{x, y}$ can be approximated as a sum of dipole $Z_{D}$ and quadrupole $Z_{Q}$ components [4,5]:

Published by the American Physical Society under the terms of the Creative Commons Attribution 3.0 License. Further distribution of this work must maintain attribution to the author(s) and the published article's title, journal citation, and DOI.

$$
\begin{aligned}
Z_{x}\left(x, x_{d}, k\right) & \cong x_{d} Z_{D x}(k)+x Z_{Q x}(k), \\
Z_{D x}(k) & \left.\equiv \frac{\partial Z_{x}}{\partial x_{d}}\right|_{x_{d}=x=0},\left.\quad Z_{Q x}(k) \equiv \frac{\partial Z_{x}}{\partial x}\right|_{x_{d}=x=0}, \\
Z_{y}\left(y, y_{d,}, k\right) & \cong y_{d} Z_{D y}(k)-y Z_{Q y}(k), \\
Z_{D y}(k) & \left.\equiv \frac{\partial Z_{y}}{\partial y_{d}}\right|_{y_{d}=y=0},\left.\quad Z_{Q y}(k) \equiv \frac{\partial Z_{y}}{\partial y}\right|_{y_{d}=y=0},
\end{aligned}
$$

where $x_{d}, y_{d}$ are the transverse coordinates of a driving particle which excites the wakefields acting on a particle with coordinates $x, y$ following the first one at a certain distance, with both particles traveling with the speed of light. This paper is focused on the theoretical and experimental study of the beam interaction with the transverse narrow-band resistive-wall impedance in the zero frequency limit, specifically the resistive-wall impedance contributed by wigglers and undulators [6] used in synchrotron light sources for x-ray generation. A formula for the multibunch current dependent tune shift induced by the resistive wall impedance has been derived by Chao, Heifets and Zotter in Ref. [4]. Their model uses the resistive wall impedance derived by Gluckstern in [7] for a cylindrical structure, multiplied by the Yokoya's coefficients given in Table I of [4] and can be found in [8]. For perfect magnetic boundary conditions, the use of Eq. (10) in Eq. (16) from [4] leads to a discrepancy with Laslett tune shift formulas [9-12]. This, together with the fact that wigglers and undulator magnets do not always satisfy the perfect magnetic boundary conditions of Laslett theory, motivated us to derive more general impedance formulas valid for arbitrary permeability. The derivation, based on the standard field matching 
technique [13-17], allows the explicit determination of the quadrupole impedance in the zero frequency limit, where the dependence on the magnetic permeability $\mu_{r}$ is given in terms of the dilogarithm function $\mathrm{Li}_{2}$ with real argument $\eta=\left(\mu_{r}-1\right) /\left(\mu_{r}+1\right)$. The paper is organized as follows. In Sec. II we describe the geometry and electromagnetic properties of the damping wigglers (DWs) and in-vacuum undulators (IVUs) for local impedance measurements used in the NSLS-II storage ring. In Sec. III we derive the complex frequency shift induced by the dipole and quadrupole impedance using the method applied in Ref. [18]. In Sec. IV we discuss the derivation of the quadrupole impedance formula of the DWs and IVUs. In Sec. V we discuss the local impedance measurements in the NSLS-II storage ring and compare them with the analytical results derived in Secs. III and IV.

\section{WIGGLERS AND UNDULATORS: GEOMETRY AND ELECTROMAGNETIC PROPERTIES}

In the NSLS-II storage ring [19], three 7 m-long damping wigglers (DWs) are installed; two of them were used for local impedance measurements. The cross section of the DW chamber with magnets located outside of the chamber is shown in Fig. 1. The aluminum vacuum chamber has an elliptical profile with inner dimensions of $2 b_{d w}=$ $11.5 \mathrm{~mm}$ (vertical) and $60 \mathrm{~mm}$ (horizontal). The thickness of the vacuum chamber at $x=0$ is $t_{d w} \approx 1 \mathrm{~mm}$, satisfying $d_{d w}>t_{d w}+b_{d w}$ and $t_{d w} \ll b_{d w}$. The chamber is nonevaporable getter (NEG) coated with a less than $1 \mu \mathrm{m}$ surface roughness. The DW magnet arrays consist of aluminumcoated neodymium-iron-boron (NdFeB) permanent magnets (relative permeability $\mu_{r}=1.05$, conductivity $\sigma_{N d}=0.6 \times 10^{6} \mathrm{~S} / \mathrm{m}$ ) and uncoated vanadium permendur soft-pole magnets $\left(\mu_{r}=400, \quad \sigma_{V P}=2.5 \times 10^{6} \mathrm{~S} / \mathrm{m}\right)$ between them, with side-clamped $\mathrm{NdFeB}$ magnets. The thickness of the aluminum coating is $\sim 20 \mu \mathrm{m}$ with conductivity of $\sigma_{A l}=31.6 \times 10^{6} \mathrm{~S} / \mathrm{m}$. The magnetic gap can be varied from $2 d_{d w}=15 \mathrm{~mm}$ to $2 d_{d w}=150 \mathrm{~mm}$. A sideview image of the DW at open position and the vacuum chamber are shown in Fig. 2.

Eight in-vacuum undulators (IVUs) are currently installed in NSLS-II; only two of them in high- $\beta_{x}$ (horizontal betatron function) were used for measurements. These devices have a complex geometry with two arrays of magnets attached to the tapered transitions from both sides [Fig. 3(a)] and enclosed in a large cylindrical vacuum

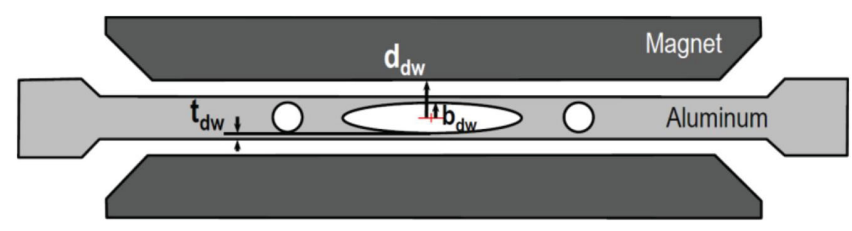

FIG. 1. Damping wiggler chamber cross section with magnetic gap closed, $d_{d w}>t_{d w}+b_{d w}$ and $t_{d w} \ll b_{d w}$.

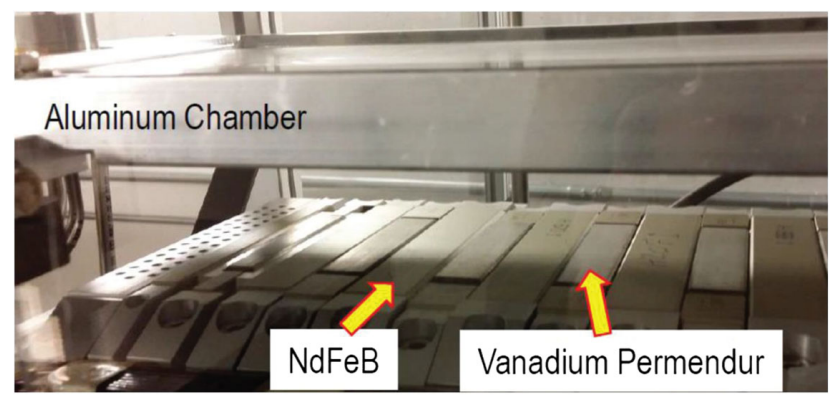

FIG. 2. Damping wiggler at open position and the aluminum vacuum chamber (side view).

vessel [Fig. 3(b)]. The magnetic poles are covered by a nickel-copper $(\mathrm{NiCu})$ foil of $t_{i}=150 \mu \mathrm{m}$ thickness $\left(t_{\mathrm{Cu}}=75 \mu \mathrm{m}, \quad \sigma_{\mathrm{Cu}}=54 \times 10^{6} \mathrm{~S} / \mathrm{m} \quad\right.$ and $t_{\mathrm{Ni}}=75 \mu \mathrm{m}$, $\left.\sigma_{\mathrm{Ni}} \approx 14 \times 10^{6} \mathrm{~S} / \mathrm{m}\right)$ to reduce the resistive-wall impedance at high frequencies, where the foil thickness is larger than the skin depth.

The difference between DW and IVU from a beam point of view is in the location of the magnet arrays. The IVU magnets are covered by $\mathrm{NiCu}$ foil and located inside the vacuum chamber [Figs. 3(a) and 3(b)], whereas the DW magnets are distributed outside the vacuum chamber (Figs. 1 and 2). A schematic magnets layout, applicable to both geometries, is shown in Fig. 4, which shows the

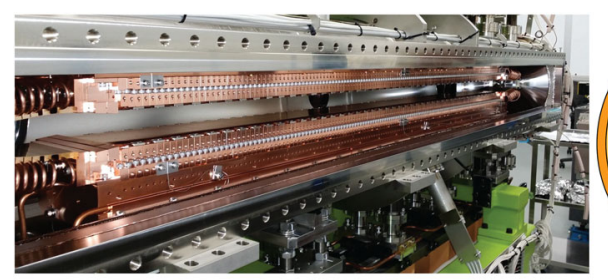

(a)

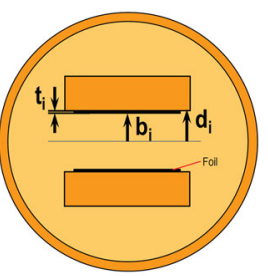

(b)
FIG. 3. (a) Image of IVU without the side cover. (b) Schematic cross section of IVU in the middle of the device. The full gap $2 b_{i}$ between the magnets can be varied from 6 to $40 \mathrm{~mm}$ for ID10 and from 5.7 to $40 \mathrm{~mm}$ for ID16.

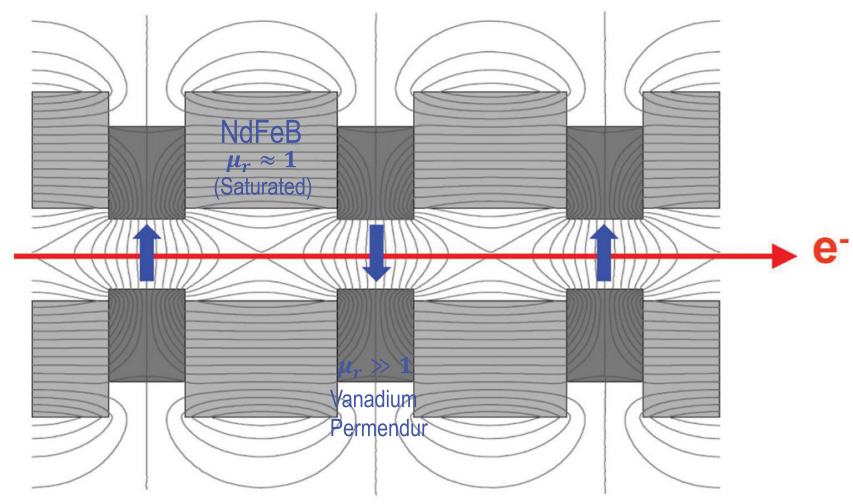

FIG. 4. Schematic layout of two magnet arrays (top and bottom) with magnetic field lines (courtesy of Clarke [6]). 
$\mathrm{NdFeB}$ permanent magnets and vanadium permendur soft-pole magnets, with magnetic field lines. Based on parameters of the magnet poles the field lines can change their direction. The length of a single $\mathrm{NdFeB}$ pole magnet, $l_{p m}$, and a single vanadium permendur pole magnet, $l_{s m}$, varies for different insertion devices (IDs). Their values are: $l_{p m}=2.65 \mathrm{~mm}, l_{s m}=8.35 \mathrm{~mm}$ for ID10 (period length $=22 \mathrm{~mm}) ; l_{p m}=3.5 \mathrm{~mm}, l_{s m}=8 \mathrm{~mm}$ for ID16 (period length $=23 \mathrm{~mm}$ ) $, l_{p m}=21 \mathrm{~mm}, l_{s m}=29 \mathrm{~mm}$ for DWs (period length $=100 \mathrm{~mm}$ ). Multiplying by the number of magnet poles of each ID we obtain the total lengths of the permanent magnet poles, $L_{p m}$, and soft-pole magnets, $L_{s m}$. Their values are: $L_{p m}=2131.5 \mathrm{~mm}$ and $L_{s m}=768.5 \mathrm{~mm}$ for ID10; $L_{p m}=1872 \mathrm{~mm}$ and
$L_{s m}=819 \mathrm{~mm}$ for ID16; $L_{p m}=2030 \mathrm{~mm}$ and $L_{s m}=$ $1470 \mathrm{~mm}$ for DWs.

\section{COMPLEX BETATRON TUNE SHIFT}

The dipole $Z_{D}$, and quadrupole $Z_{Q}$, impedances defined by Eqs. (1) and (2) give rise to potential beam instabilities. We derive the complex frequency shift induced by the dipole and quadrupole impedance on a uniform bunch train of $M$ bunches each populated with $N$ particles, using the methods developed in [18]. Without loss of generality, we restrict the analysis to the horizontal plane.

Let us consider the set of $M$-coupled Vlasov equations governing the evolution of the phase density $\Psi_{m}$ of the $m$ th bunch $(m=0, \ldots, M-1)$,

$$
\begin{aligned}
& \frac{\partial \Psi_{m}}{\partial t}-\eta c \delta \frac{\partial \Psi_{m}}{\partial z}+\frac{\omega_{s}^{2}}{\eta c} z \frac{\partial \Psi_{m}}{\partial \delta}+p_{x} \frac{\partial \Psi_{m}}{\partial x}-\omega_{\beta}^{2} x \frac{\partial \Psi_{m}}{\partial p_{x}} \\
& -A\left[\sum_{m^{\prime}=0}^{M-1} \sum_{k=0}^{+\infty} \int_{-\infty}^{z} d z^{\prime} W_{D x}\left(z-z^{\prime}+a_{m^{\prime} m}^{k} C\right) \int_{-\infty}^{+\infty} d x^{\prime} x^{\prime} \rho_{m^{\prime}}\left(z^{\prime}, x^{\prime}, t-a_{m^{\prime} m}^{k} T_{0}\right)\right] \frac{\partial \Psi_{m}}{\partial p_{x}} \\
& -x A\left[\sum_{m^{\prime}=0}^{M-1} \sum_{k=0}^{+\infty} \int_{-\infty}^{z} d z^{\prime} W_{Q x}\left(z-z^{\prime}+a_{m m^{\prime}}^{k} C\right) \lambda_{m^{\prime}}\left(z^{\prime}, x^{\prime}, t-a_{m^{\prime} m}^{k} T_{0}\right)\right] \frac{\partial \Psi_{m}}{\partial p_{x}}=0
\end{aligned}
$$

where $\quad a_{m m^{\prime}}^{k}=k+\frac{m^{\prime}-m}{M}, \quad A=\frac{N r_{0} c}{\left(\gamma T_{0}\right)}, \quad \rho_{m}(z, x, t)=$ $\int d \delta d p_{x} \Psi_{m}\left(z, \delta, x, p_{x}, t\right)$ and $\lambda_{m}(z, t)=\int d x \rho_{m}(z, x, t)$. Here the phase space coordinates $\left(z, \delta, x, p_{x}\right)$ are $z=c \tau$, where $\tau$ is arrival time, $\delta=\left(E-E_{0}\right) / E_{0}$, where $E_{0}$ is the energy of the reference particle in electron volts, transverse position $x$ and transverse momentum $p_{x} ; C$ is the ring circumference, $T_{0}$ the revolution period, $\eta$ is the slippage factor, $\omega_{s}=2 \pi v_{s} / T_{0}$ where $v_{s}$ is the synchrotron tune, $\omega_{\beta}=2 \pi v_{x} / T_{0}$ where $v_{x}$ is the horizontal betatron tune, $r_{0}$ is the classical electron radius, $N$ the number of particles in the bunch, $\gamma$ the Lorentz factor, and $c$ is the speed of light. $W_{D, Q x}$ is the horizontal dipole/quadrupole wake function and is related to the horizontal dipole/ quadrupole impedance by the Fourier transform pair

$$
\begin{gathered}
W_{D, Q x}(z)=-i \frac{c}{2 \pi} \int_{-\infty}^{+\infty} d k e^{i k z} Z_{D, Q x}(k), \\
Z_{D, Q x}(k)=\frac{i}{c} \int_{-\infty}^{+\infty} d z e^{-i k z} W_{D, Q x}(z) .
\end{gathered}
$$

Multiplying Eq. (3) by $x$ first and by $p_{x}$ second, integrating by parts we obtain the equations for the evolution of the dipole moments $\left\langle x_{m}\right\rangle=\int d z d \delta d x d p_{x} x \Psi_{m}\left(z, \delta, x, p_{x}, t\right)$ and $\left\langle p_{x_{m}}\right\rangle=\int d z d \delta d x d p_{x} p_{x} \Psi_{m}\left(z, \delta, x, p_{x}, t\right)$. The equation for $\left\langle x_{m}\right\rangle$ reads

$$
\begin{aligned}
\frac{d}{d t}\left\langle x_{m}\right\rangle & =\int d z d \delta d x d p_{x} x \Psi_{m}\left(z, \delta, x, p_{x}, t\right) \\
& =-\int d z d \delta d p_{x} p_{x}\left(x \Psi_{m} \mid \begin{array}{l}
x=+\infty \\
x=-\infty
\end{array}-\int d x \Psi_{m}\right) \\
& =p_{x_{m}},
\end{aligned}
$$

where the range of integration is intended from $-\infty$ to $+\infty$ when omitted. For $p_{x_{m}}$ we proceed similarly obtaining the result

$$
\begin{aligned}
& \frac{d^{2}}{d t^{2}}\left\langle x_{m}\right\rangle+\omega_{\beta}^{2}\left\langle x_{m}\right\rangle \\
& =+A \sum_{m^{\prime}=0}^{M-1} \sum_{k=0}^{+\infty} \int d z \int d z^{\prime} W_{D x}\left(z-z^{\prime}+a_{m^{\prime} m}^{k} C\right) \lambda_{m^{\prime}}(z, t) \\
& \quad \times \int d x^{\prime} x^{\prime} \rho_{m^{\prime}}\left(z^{\prime}, x^{\prime}, t-a_{m^{\prime} m}^{k} T_{0}\right) \\
& \quad+A \sum_{m^{\prime}=0}^{M-1} \sum_{k=0}^{+\infty} \int d z \int d z^{\prime} W_{Q x}\left(z-z^{\prime}+a_{m^{\prime} m}^{k} C\right) \lambda_{m \prime} \\
& \quad \times\left(z, t-a_{m^{\prime} m}^{k} T_{0}\right) \int d x^{\prime} x^{\prime} \rho_{m}\left(z^{\prime}, x^{\prime}, t\right),
\end{aligned}
$$

where the upper limit of integration in $z^{\prime}$ has been extended to $+\infty$ using the causality property of $W_{D, Q x}\left(W_{D, Q x}(z)=0\right.$ if $z<0)$. Using the approximation $\rho_{m}(z, x, t)=\lambda(z) \mu_{m}(x, t)$, 
where $\mu_{m}(x, t)=\int d z \rho_{m}(z, x, t)$ is the transverse distribution density of the $m$ th bunch, we have

$$
\begin{aligned}
\frac{d^{2}}{d t^{2}}\left\langle x_{m}\right\rangle+\omega_{\beta}^{2}\left\langle x_{m}\right\rangle= & A \sum_{m^{\prime}=0}^{M-1} \sum_{k=0}^{+\infty} f_{D}\left(a_{m^{\prime} m}^{k} C\right)\left\langle x_{m^{\prime}}\left(t-a_{m^{\prime} m}^{k} T_{0}\right)\right\rangle \\
& +A \sum_{m^{\prime}=0}^{M-1} \sum_{k=0}^{+\infty} f_{Q}\left(a_{m^{\prime} m}^{k} C\right)\left\langle x_{m}\right\rangle
\end{aligned}
$$

where $f_{D, Q}(x) \equiv \int d z \int d z^{\prime} W_{D, Q x}\left(z-z^{\prime}+x\right) \lambda(z) \lambda\left(z^{\prime}\right)$.

A similar derivation leads to the vertical equations of motion. The first term on the rhs of Eq. (8) leads to the complex frequency shift of bunch mode $\mu$ driven by the dipole impedance [see for example Eq. (B6) of [18]]. The second term on the rhs of Eq. (8) is due to the quadrupole impedance. The evaluation of the coefficient of $\left\langle x_{m}\right\rangle$ reads

$$
\begin{aligned}
A \sum_{m^{\prime}=0}^{M-1} \sum_{k=0}^{+\infty} f\left(a_{m m^{\prime}}^{k} C\right) & =A \sum_{k=0}^{+\infty} f\left(k \frac{C}{M}\right) \\
& =-i \frac{A}{2 \pi} \int_{-\infty}^{+\infty} d \omega|\tilde{\lambda}(\omega)|^{2} Z_{Q x}(\omega) \sum_{k=-\infty}^{+\infty} e^{i k \frac{\omega T_{0}}{M}}
\end{aligned}
$$

where we used Eq. (4) with the change of variable $\omega=k c$. Using the identity

$$
\sum_{k=-\infty}^{+\infty} e^{i k z}=2 \pi \sum_{p=-\infty}^{+\infty} \delta(z-2 \pi p),
$$

it follows

$$
\begin{aligned}
& A \sum_{m^{\prime}=0}^{M-1} \sum_{k=0}^{+\infty} f\left(a_{m m^{\prime}}^{k} C\right) \\
& =-i A \sum_{p=-\infty}^{+\infty} \int_{-\infty}^{+\infty} d \omega|\tilde{\lambda}(\omega)|^{2} Z_{Q x}(\omega) \delta\left(\frac{\omega T_{0}}{M}-2 \pi p\right) \\
& =-i A \frac{M}{T_{0}} \sum_{p=-\infty}^{+\infty}\left|\tilde{\lambda}\left(p M \omega_{0}\right)\right|^{2} Z_{Q x}\left(p M \omega_{0}\right) .
\end{aligned}
$$

Assuming for Eq. (8) a solution of the form $\left\langle x_{m}\right\rangle=a_{m} e^{-i\left(\omega_{\beta}+\Omega\right) t}$, where $\Omega \in \mathbb{C}$ and $|\Omega| \ll \omega_{\beta}$, by neglecting the contribution from the dipole impedance the complex betatron frequency shift is solely induced by the quadrupole impedance

$$
\Omega=-i A \frac{M}{T_{0}} \sum_{p=-\infty}^{+\infty}\left|\tilde{\lambda}\left(p M \omega_{0}\right)\right|^{2} Z_{Q x}\left(p M \omega_{0}\right)
$$

Using the condition $Z_{Q x}^{*}(\omega)=-Z_{Q x}(-\omega)$, which follows from the reality of the wake functions, and defining the betatron tune shift $\Delta \nu_{x} \equiv \operatorname{Im} \Omega$, from Eq. (11) it follows

$$
\begin{aligned}
\Delta \nu_{x}= & |\tilde{\lambda}(0)|^{2} A \frac{M}{T_{0}} \operatorname{Im} Z_{Q x}(0) \\
& +2 A \frac{M}{T_{0}} \sum_{p=1}^{+\infty}\left|\tilde{\lambda}\left(p M \omega_{0}\right)\right|^{2} \operatorname{Im} Z_{Q x}\left(p M \omega_{0}\right) .
\end{aligned}
$$

Therefore the quadrupole impedance, being the complex frequency shift purely imaginary, does not induce any growth in the amplitude of betatron motion, just a change in the oscillation frequency.

We notice here that the terms with $p \geq 1$ contribute at frequencies higher than the angular revolution frequency $\omega_{0}$, with the first contribution coming from the impedance sampled at the $M \omega_{0}$. For $M \gg 1$, at frequencies $\omega \geq M \omega_{0}$ the impedance decreases as $1 / \sqrt{\omega}$. Assuming $\operatorname{Im} Z_{Q x}$ monotically decreasing at all frequencies, the main contribution to the tune shift comes from the quadrupole impedance sampled at zero frequency.

\section{QUADRUPOLE IMPEDANCE}

Both geometries, DW and IVU, can be analytically approximated by a multiparallel-plates model, where permanent and soft-pole magnets are modeled with different magnetic boundary conditions, and the total impedance is determined by adding all the contributions neglecting transient effects at the transition region between permanent and soft-pole magnets. Given the lengths of the magnet components discussed at the end of Sec. II, we believe transient effects to be of second order.

A schematic layout of the parallel-plates model is shown in Fig. 5. Region $I$ represents the region inside the metallic walls (region II), which correspond to the vacuum chamber for the DWs and the $\mathrm{NiCu}$ foil for the IVUs. The resistive walls have thickness $t$ and full gap $2 b$. Region $I I I$, with gap $s$ and in which we assume vacuum, represents the region between the metallic walls and magnets (region $I V$ ). In the IVU case, the $\mathrm{NiCu}$ foil is attached to the magnets, thus $s=0$. The magnets are assumed to have infinite thickness.

We will calculate the quadrupole impedance per unit length assuming that the driving particle is equidistant from the plates, at $x_{d}=y_{d}=0$, and we will use Gaussian units.

We will start from Maxwell's equations and analyze them in Fourier domain ending up with general equations

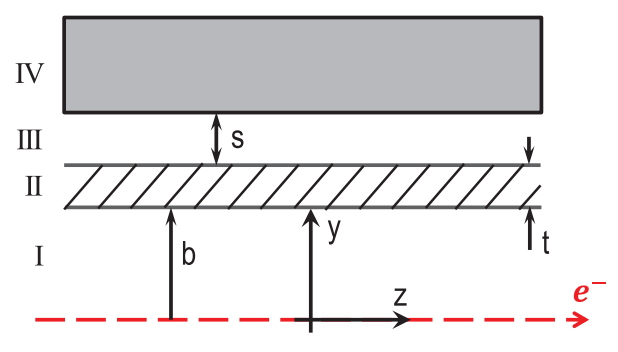

FIG. 5. Geometry of the vacuum chamber in planar approximation. 
valid for arbitrary frequencies. We use the following Maxwell's equations:

$$
\nabla \times \vec{E}=-\frac{\mu_{r}}{c} \frac{\partial \vec{H}}{\partial t}, \quad \nabla \times \vec{H}=\frac{4 \pi}{c} \vec{J}+\frac{\varepsilon_{r}}{c} \frac{\partial \vec{E}}{\partial t},
$$

where in region $I(|y|<b)$ we assume a point particle source moving in the longitudinal direction $z$ at the speed of light, thus $\vec{J}=\left(J_{z}, 0,0\right)$, where $J_{z}=I_{\omega} \delta(x) \delta(y) \delta(z-c t)$, in region II $(b<|y|<b+t)$ and in region IV $(|y|>b+t+s)$ we assume $\vec{J}=\sigma_{c} \vec{E}$ (Ohm's Law) and in region III $(b+t<|y|<b+t+s)$ we assume $\vec{J}=0$. Here $\varepsilon_{r}$ is the relative electric permittivity and $\mu_{r}$ the relative magnetic permeability. With the following Fourier transformation pair,

$$
\begin{gathered}
\hat{\vec{F}}(k, q, y, t)=\int_{-\infty}^{\infty} d z \int_{-\infty}^{\infty} d x e^{-i k z-i q x} \vec{F}(z, x, y, t), \\
\vec{F}(z, x, y, t)=\frac{1}{(2 \pi)^{2}} \int_{-\infty}^{\infty} d k \int_{-\infty}^{\infty} d q e^{i k z+i q x} \hat{\vec{F}}(k, q, y, t),
\end{gathered}
$$

where $\vec{F}=(\vec{E}, \vec{H}, \vec{J})$, it follows that $\tilde{J}_{z}=I_{\omega} \delta(y) e^{-i k c t}$, thus we look for a solution of the form $\hat{\vec{F}}(k, q, y, t)=$ $\tilde{\vec{F}}(k, q, y) e^{-i k c t}$. To simplify the analysis, we rescale $\tilde{\vec{F}}$ according to $\tilde{\vec{F}} \rightarrow \tilde{\vec{F}} c /\left(2 \pi I_{\omega}\right)$. With an abuse of notation, we retain the tilde symbol. Using $\bar{E}_{z} \equiv \tilde{E}_{z} / k, \bar{H}_{z} \equiv \tilde{H}_{z} / k$ and ${ }^{\prime} \equiv \partial / \partial y$, from Eq. (13) we obtain, in regions $I$ and $I I I$

$$
\begin{aligned}
\bar{E}_{z}^{\prime} & =i q \bar{H}_{z}, \quad \bar{H}_{z}^{\prime}=-i q \bar{E}_{z}, \\
\tilde{E}_{x} & =\tilde{H}_{y}+q \bar{E}_{z}, \quad \tilde{H}_{x}=-\tilde{E}_{y}+q \bar{H}_{z}, \\
i q \tilde{E}_{y}-\bar{H}_{y}^{\prime} & =i \bar{H}_{z}\left(q^{2}+k^{2}\right), \\
i q \tilde{H}_{y}+\bar{E}_{z}^{\prime} & =-i \bar{E}_{z}\left(q^{2}+k^{2}\right)+\alpha \delta(y),
\end{aligned}
$$

where $\alpha=2$ in region $I$ and $\alpha=0$ in region $I I I$, and in regions $I I$ and $I V$

$$
\begin{aligned}
\bar{E}_{z}^{\prime \prime} & =\left[q^{2}-k^{2}\left(\varepsilon_{r} \mu_{r}-1\right)-i k \xi \mu_{r}\right] \bar{E}_{z}, \\
\bar{H}_{z}^{\prime \prime} & =\left[q^{2}-k^{2}\left(\varepsilon_{r} \mu_{r}-1\right)-i k \xi \mu_{r}\right] \bar{H}_{z}, \\
\tilde{E}_{x} & =\frac{k\left(q \bar{E}_{z}-i \mu_{r} \bar{H}_{z}^{\prime}\right)}{k\left(1-\varepsilon_{r} \mu_{r}\right)-i \xi \mu_{r}}, \\
\tilde{E}_{y} & =-\frac{k\left(q \mu_{r} \bar{H}_{z}+i \bar{E}_{z}^{\prime}\right)}{k\left(1-\varepsilon_{r} \mu_{r}\right)-i \xi \mu_{r}} \\
\tilde{H}_{x} & =\frac{q k \bar{H}_{z}-\left(\xi-i \varepsilon_{r} k\right) \bar{E}_{z}^{\prime}}{k\left(1-\varepsilon_{r} \mu_{r}\right)-i \xi \mu_{r}} \\
\tilde{H}_{y} & =\frac{i\left[q\left(\xi-i \varepsilon_{r} k\right) \bar{E}_{z}-k \bar{H}_{z}^{\prime}\right]}{k\left(1-\varepsilon_{r} \mu_{r}\right)-i \xi \mu_{r}}
\end{aligned}
$$

where $\xi=4 \pi \sigma_{c} / c$. Due to the symmetry of the problem it is sufficient to consider the equations in the region of positive $y$ only. As it follows from Eq. (14), the electric field $E_{z}$ satisfies the equation $\bar{E}_{z}^{\prime \prime}-q^{2} \bar{E}_{z}=0$. Due to the symmetry of the problem we seek $\bar{E}_{z}$ in region I in the form

$$
\bar{E}_{z}=E_{0}(q, k) \cosh q y .
$$

When the function $E_{0}(q, k)$ is known, the horizontal and vertical quadrupole impedances have opposite sign and are given by the following formula:

$$
Z_{Q x, y}(k)= \pm \frac{1}{c} \int_{-\infty}^{\infty} E_{0}(q, k) q^{2} d q .
$$

The above equations are valid for arbitrary frequencies, and are well posed to be studied in the zero frequency limit. To this end, we calculate the quadrupole impedance at zero frequency by evaluating Eqs. (14) and (15) at $k=0$, and solve them by imposing matching conditions on the tangential field components at the boundaries of the different regions. Since only the tangential field components are used, the solutions we need are, in region $I$

$$
\left(\begin{array}{cc}
\bar{E}_{z} & \bar{H}_{z} \\
\tilde{E}_{x} & \tilde{H}_{x}
\end{array}\right)=\left(\begin{array}{cc}
E_{0} & 0 \\
i B_{1} & -1
\end{array}\right) \cosh q y+i\left(\begin{array}{cc}
0 & -E_{0} \\
1 & A_{1}
\end{array}\right) \sinh q y,
$$

where $E_{0}=\left(-A_{1}+i B_{1}\right) / q$, in regions $I I$ and $I V$

$$
\left(\begin{array}{cc}
\bar{E}_{z} & \bar{H}_{z} \\
\tilde{E}_{x} & \mu_{r} \tilde{H}_{x}
\end{array}\right)=\left(\begin{array}{cc}
E_{n s} & H_{n s} \\
0 & i q E_{n s}
\end{array}\right) e^{-q y}+\left(\begin{array}{cc}
E_{n c} & H_{n c} \\
0 & -i q E_{n c}
\end{array}\right) e^{q y},
$$

where $n=2,4$, and in region III

$\left(\begin{array}{cc}\bar{E}_{z} & \bar{H}_{z} \\ \tilde{E}_{x} & \tilde{H}_{x}\end{array}\right)=\left(\begin{array}{cc}E_{3} & i E_{3} \\ i B_{3} & -A_{3}\end{array}\right) e^{-q y}+\left(\begin{array}{cc}E_{4} & -i E_{4} \\ i B_{4} & i A_{4}\end{array}\right) e^{q y}$,

where $E_{3}=-\left(A_{3}+i B_{3}\right) / q$, and $E_{4}=\left(-A_{4}+i B_{4}\right) / q$. The unknowns $A_{1}, B_{1}, E_{2 s}, E_{2 c}, H_{2 s}, H_{2 c}, A_{3}, B_{3}, A_{4}$, $B_{4}, E_{4 s}, E_{4 c}, H_{4 s}, H_{4 c}$ are determined by field matching conditions, which consist of the continuity of the tangential field components at the interfaces of the different regions,

$$
\begin{aligned}
& \mathbb{E}^{I}(b)=\mathbb{E}^{I I}(0), \quad \mathbb{Q}^{I}(b)=\mathbb{Q}^{I I}(0), \\
& \mathbb{E}^{I I}(t)=\mathbb{E}^{I I I}(0), \quad \mathbb{\boxplus}^{I I}(t)=\mathbb{\square}^{I I I}(0), \\
& \mathbb{E}^{I I I}(s)=\mathbb{E}^{I V}(0), \quad \mathbb{\mathbb { Q } ^ { I I I }}(s)=\mathbb{\boxplus}^{I V}(0),
\end{aligned}
$$

where $\mathbb{E}=\left(\bar{E}_{z}, \tilde{E}_{x}\right)$ and $\mathbb{U}=\left(\bar{H}_{z}, \tilde{H}_{x}\right)$, and by the additional condition that the field components vanish at infinity. Assuming the thickness of the magnets to be infinite, the 
latter condition imposes that $E_{4 c}=H_{4 c}=0$, thus leading to a system of twelve equations in twelve unknowns. We now solve the linear system of equations assuming that $\varepsilon_{r}$ is equal to one in all regions, and that is equal to one in all regions other than region IV. The solution is straightforward. Here we give the values of $A_{1}, B_{1}$ which determine $E_{0}=\left(-A_{1}+i B_{1}\right) / q$

$$
A_{1}=-i \frac{\left(1+\mu_{r} \operatorname{coth} q d\right)}{\operatorname{coth} q d+\mu_{r}}, \quad B_{1}=-\tanh q b,
$$

where $d=b+t+s$ is the distance from the center of the vacuum chamber to the inner surface of the magnet (halfmagnetic gap). From Eq. (17), the horizontal and vertical quadrupole impedance at zero frequency reads

$$
\begin{aligned}
& Z_{Q x, y}(0)= \pm i \frac{2}{c} \int_{0}^{\infty} q\left(\frac{1+\mu_{r} \operatorname{coth} q d}{\operatorname{coth} q d+\mu_{r}}-\tanh q b\right) d q \\
& = \pm i \frac{2}{c} \int_{0}^{\infty} q\left(\frac{1+\mu_{r} \operatorname{coth} q d}{\operatorname{coth} q d+\mu_{r}}-1\right) d q \pm i \frac{2}{c} \int_{0}^{\infty} q(1-\tanh q b) d q \\
& = \pm i \frac{4}{c} \int_{0}^{\infty} \frac{q}{e^{2 q d} / \eta-1} d q \pm i \frac{\pi^{2}}{12 c b^{2}}
\end{aligned}
$$

where

$$
\eta=\frac{\mu_{r}-1}{\mu_{r}+1} .
$$

From

$$
\int_{0}^{\infty} \frac{q}{e^{2 q d} / \eta-1} d q=\frac{4}{d^{2}} \operatorname{Li}_{2}(\eta),
$$

where $\operatorname{Li}_{2}(z)$ is the dilogarithm defined as

$$
\operatorname{Li}_{2}(z)=\sum_{k=1}^{\infty} \frac{z^{k}}{k^{2}}, \quad z \in \mathbb{C},
$$

the general equation of the quadrupole impedance at zero frequency, taking into account the general magnetic properties of the magnets, reads

$$
Z_{Q x, y}(0)= \pm i \frac{\pi^{2}}{12 c b^{2}}\left(1+2 \frac{b^{2}}{d^{2}} \mathrm{f}(\eta)\right),
$$

where

$$
\mathrm{f}(\eta)=\frac{6}{\pi^{2}} \mathrm{Li}_{2}(\eta)
$$

Here we point out that Eq. (22) is equivalent to Eq. (11) of Ref. [16] evaluated at zero frequency for $s=0$, derived using a different approach. We notice that in the limit $\mu_{r} \rightarrow \infty$ (perfect magnets) $\mathrm{f}(\eta)=1$, while for $\mu_{r}=1$ (saturated magnets) $\mathrm{f}(\eta)=0$. For the soft magnetic poles $\mu_{r}=400$, implying $\mathrm{f}(\eta)=0.99$, while for the permanent magnets $\mu_{r}=1.05$, implying $\mathrm{f}(\eta)=0.001$. Equation (24) gives the impedance per unit length in CGS units, to convert the impedance into SI units, multiply by $c Z_{0} / 4 \pi$. The vertical, $\Delta \nu_{y}$, or horizontal, $\Delta \nu_{x}$, tune shift induced by the quadrupole impedance of DWs or IVUs at zero frequency is given, in the point-bunch approximation, by Eq. (12) neglecting the contribution from the terms with $p \geq 1$,

$$
\Delta \nu_{x, y}=\frac{I_{a v} L}{4 \pi E / e} \beta_{x, y} \operatorname{Im} Z_{Q x, y}(0),
$$

where $c / \omega_{\beta}$ is replaced by the local beta function $\beta_{x, y}, I_{a v}$ is the average current and $E$ is the energy of the beam, $L$ is the length of the impedance structure and the quadrupole impedance is given by Eq. (24). The well-known Laslett formula [20] for the incoherent tune shift,

$$
\begin{aligned}
\Delta \nu_{x, y} & =-\frac{N M r_{e} \beta_{x}}{\pi \gamma}\left(\frac{\epsilon_{1}^{H, V}}{b^{2}}+\frac{\epsilon_{2}^{H, V}}{d^{2}}\right) \frac{L}{C}, \\
\epsilon_{1}^{H, V} & = \pm \frac{\pi^{2}}{48}, \quad \epsilon_{2}^{H, V}= \pm \frac{\pi^{2}}{24},
\end{aligned}
$$

is a particular case of Eq. (25) with the impedance given by Eq. (24) for a perfect magnet. Here $\epsilon_{1}^{H, V}$ and $\epsilon_{2}^{H, V}$ are the incoherent Laslett electric and magnetic image coefficients respectively. The quadrupole impedance, calculated from Eq. (24), can be written for IVUs and DWs in the following form as a function of half-magnetic gap $d$ :

$$
\begin{array}{r}
Z_{Q x, y}^{I V U}(d)= \pm i \frac{\pi^{2}}{12 c(d-t)^{2}}\left(1+2 \frac{(d-t)^{2}}{d^{2}} \mathrm{f}(\eta)\right) \\
Z_{Q x, y}^{D W}(d)= \pm i \frac{\pi^{2}}{12 c b^{2}}\left(1+\frac{2 b^{2}}{d^{2}} \mathrm{f}(\eta)\right) .
\end{array}
$$

The difference between Eq. (27) and (28) is the presence of the vacuum chamber with fixed aperture for DW, while in the IVU geometry the metal layer (nickel-copper foil) is attached to the magnets.

\section{MEASUREMENTS}

For beam impedance measurements, we used 2DWs (DW18 and DW28) and 2IVUs (ID10 and ID16). The beta functions are $\beta_{x / y}=21 \mathrm{~m} / 5.1 \mathrm{~m}$ and $\beta_{x / y}=20.5 \mathrm{~m} / 3.6 \mathrm{~m}$ at the location of DWs and IVUs respectively. The electron energy in the NSLS-II storage ring is $E=3 \mathrm{GeV}$. The vertical $\nu_{y}$, and horizontal $\nu_{x}$, betatron tunes have been measured as a function of average current $I_{a v}$ uniformly distributed in 1080 bunches. During the measurements, coherent betatron oscillations of small amplitude have been 

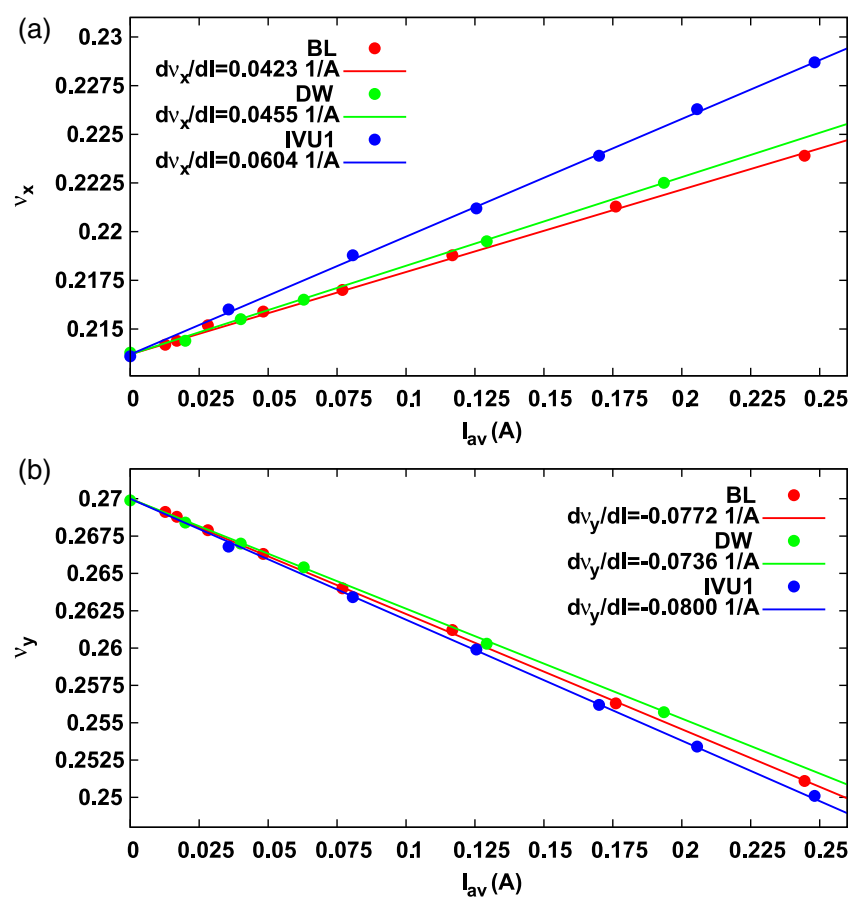

FIG. 6. Horizontal (a) and vertical (b) betatron tune shifts versus average current for lattices with local $\beta_{x}=21 \mathrm{~m}$.

excited independently in both planes with the use of pinger magnets. The contribution from the dipole impedance to the betatron tune shifts has been studied with a series of measurements by lowering the fractional tune, and found to be negligible, implying that we can assume that the contribution to the betatron tune shifts is solely due to the quadrupole impedance.

Four lattice configurations have been studied: Bare lattice (BL) - with all DW and IVU gaps open; the lattice with 2DW gaps closed (DW), the lattice with 2IVU gaps closed (IVU1), and the lattice with 2IVU gaps partially closed (IVU2). The measured betatron tunes $\nu_{x}$ and $\nu_{y}$ versus $I_{a v}$ are shown in Fig. 6 (dots), where the tune slopes $d \nu_{x} / d I_{a v}$ and $d \nu_{y} / d I_{a v}$, obtained by linear fitting of the measured data, are shown as solid lines. According to Eqs. (24) and (25), the vertical tune slope is negative while the horizontal tune slope is positive. Closing the magnetic gaps of 2DWs or 2IVUs separately, allows us to measure the difference in tune slopes,

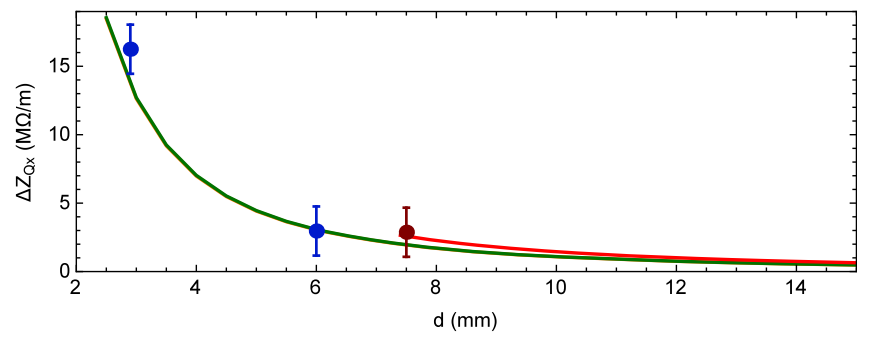

FIG. 7. Summary results of the quadrupole impedance vs magnetic gap $d$ for IVU and DW. Blue dots and green solid line are measured data vs analytically obtained by Eq. (29) for the IVU case. Dark red dot and red solid line are for one DW.

$$
\Delta_{x, y}=\sum\left(\left.\frac{d \nu_{x, y}}{d I_{a v}}\right|_{D W, I V U}-\left.\frac{d \nu_{x, y}}{d I_{a v}}\right|_{B L}\right)
$$

where the summation is done over the contributions from DWs, IVU10 and IVU16 (see Table I), and estimate the measured local quadrupole impedance at zero frequency (Fig. 7, blue and dark red dots) using Eqs. (27) and (28).

Comparisons of measured $\Delta_{x, y}^{m}$ and theoretical $\Delta_{x, y}^{t}$ data are shown in Table I. The results are in a good agreement for the horizontal tune slope differences, while the vertical tune slopes show some discrepancy as in the case of DW. Here the vertical beta function is significantly smaller than the horizontal one and the vertical measurements become less sensitive to changes. Since the quadrupole impedance is the same for both planes, except the sign [Eq. (24)], we focused on reliable measurements of the horizontal betatron tune change. The accuracy of the betatron tune measurements from Fourier analysis of the betatron oscillations is $\sim 2 \times 10^{-4}$ [21], while the accuracy we estimated from linear fitting of the measured data is $\sim 0.001$. Possible sources of enhanced error during the measurements can be fluctuations in the power supplies and uncertainty in measurements of the average beam current.

The quadrupole impedances, obtained from the measured differences in tune slopes $\Delta_{x, y}^{m}$ (Table I) agree well with analytically derived data. Figure 7 shows the difference in impedances with open and closed gaps. The solid lines represent the analytical results of Eq. (27) for IVU (green line) and of Eq. (28) for DW (red line) and dots are the measured data for each individual component (IVU_blue dots, DW-dark red dot). In the case of

TABLE I. Comparison of the differences in tune slopes, measured $\Delta_{x, y}^{m}$ vs theoretical $\Delta_{x, y}^{t}$ for lattices with $\beta_{x / y}=21 \mathrm{~m} / 5.1 \mathrm{~m}$ (DW) and $\beta_{x / y}=21 \mathrm{~m} / 3.6 \mathrm{~m}$ (IVUs), where $d$ is a half-magnetic gap.

\begin{tabular}{lccccccc}
\hline \hline Lattice & DW & ID10 & ID16 & $\begin{array}{c}\Delta_{x}^{m}, A^{-1} \\
\text { Measurement }\end{array}$ & $\begin{array}{c}\Delta_{x}^{t}, A^{-1} \\
\text { Theory }\end{array}$ & $\begin{array}{c}\Delta_{y}^{m}, A^{-1} \\
\text { Measurement }\end{array}$ & $\begin{array}{c}\Delta_{y}^{t}, A^{-1} \\
\text { Theory }\end{array}$ \\
\hline BL & 75 & 20 & 20 & $\ldots$ & $\ldots$ & $\ldots$ & $\ldots$ \\
DW & 7.5 & 20 & 20 & 0.0032 & 0.0028 & 0.0044 & -0.0007 \\
IVU1 & 75 & 3 & 2.85 & 0.0181 & 0.0146 & -0.0028 & -0.0026 \\
IVU2 & 75 & 6 & 6 & 0.0033 & 0.0031 & -0.0010 & -0.0006 \\
\hline \hline
\end{tabular}



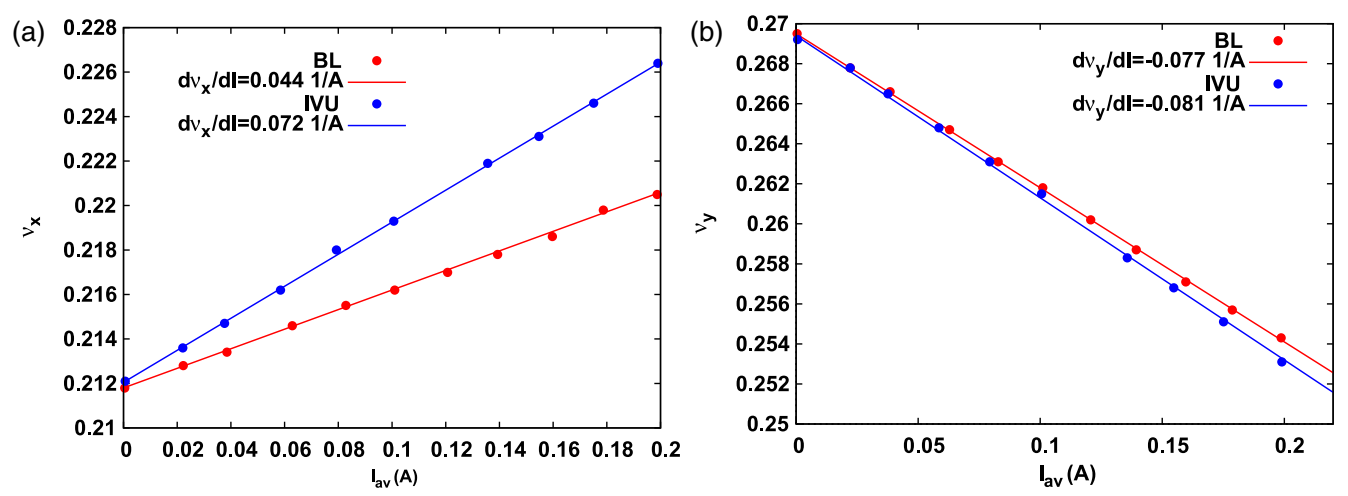

FIG. 8. Horizontal (a) and vertical (b) betatron tune shifts versus average current for lattices with local $\beta_{x}$-bumps in IDs location, where the local beta functions $\beta_{x}=31 \mathrm{~m}$ and $\beta_{y}=3.6 \mathrm{~m}$ (IVUs).

IVU we were able to compare the results for two different magnet gaps, 3 and $6 \mathrm{~mm}$.

The tune slopes behavior has been observed to be independent of the bunch filling pattern [22], which excludes ion related effects. The betatron tune shift dependence on multibunch intensity can be amplified by increasing the local betatron function. The magnet lattice of NSLS-II is quite flexible, since all quadrupoles have individual power supplies. To study effects of the local beta functions dependence on the tune slopes, we were able to create local bumps in the horizontal beta function $\beta_{x}$ in two straight sections with IVUs (ID10 and ID16). Perturbations of beta functions outside these two cells were rather small. The measured difference in tune slopes for the two IVUs with a $3 \mathrm{~mm}$ magnetic gap and local beta bump $\beta_{x}=31 \mathrm{~m}$ (instead of $\beta_{x}=21 \mathrm{~m}$ as designed) is $\Delta_{x, y}=+0.028 /$ $-0.004 \mathrm{~A}^{-1}$ (Fig. 8). These values scale down well, as expected, to the values presented in Table I for IVU1 by applying the ratio between the two local beta functions. The single-bunch contribution to the horizontal tune shifts due to change in intensity has been studied separately in NSLS-II and has been shown to have a negligible effect up to $1 \mathrm{~mA}$ per bunch [21].

\section{CONCLUSIONS}

We have presented general equations describing the impedance for arbitrary frequencies, and arbitrary electric permittivity and magnetic permeability. An explicit formula, Eq. (24), has been derived for the quadrupole impedance at zero frequency including the general magnetic properties of the magnets. In the particular case of the perfect magnets Eq. (24) reduces to the incoherent tune shift formula of Laslett. The application of Eq. (24) to wigglers and undulators has led to Eqs. (27) and (28). The explicit dependence of the quadrupole impedance on the magnetic gap has been found to be in a good agreement with the measurements. A local $\beta_{x}$-bump has been applied in the two IVU (ID10 and ID16) locations to cross-check the tune slope dependence. As a result, the measured tune slope has been found to be linear and proportional to the local beta function, as expected.

\section{ACKNOWLEDGMENTS}

This work was supported by Department of Energy Contract No. DE-AC02-98CH10886. The work by G. Stupakov was supported by Department of Energy Contract No. DE-AC03-76SF00515. We thank E. Zitvogel, G. Wang and T. Shaftan for their help during beam studies and useful discussions, and C. Kitegi, H. Fernandes, O. Chubar, J. Rank and T. Tanabe for their support with clarification of the geometric dimensions and electrodynamics properties of IDs magnets.

[1] A. W. Chao, Physics of Collective Beam Instabilities in High Energy Accelerators (John Wiley \& Sons, New York, 1993).

[2] K. Y. Ng, Physics of Intensity Dependent beam Instabilities (World Scientific Publishing, Singapore, 2006).

[3] B. W. Zotter and S. A. Kheifets, Impedances and Wakes in High-Energy Particle Accelerators (World Scientific, London, 1998).

[4] A. Chao, S. Heifets, and B. Zotter, Tune shifts of bunch trains due to resistive vacuum chambers without circular symmetry, Phys. Rev. ST Accel. Beams 5, 111001 (2002).

[5] S. Heifets, A. Wagner, and B. Zotter, SLAC, Report No. SLAC/AP 110, 1998.

[6] J. A. Clarke, The Science and Technology of Undulators and Wigglers (Oxford Science Publications, New York, 2004).

[7] R. Gluckstern, CERN Report No. 2000-011.

[8] S. Heifets, SLAC Report No. SLAC-PUB-7985, 1998.

[9] K. Y. Ng, FERMILAB, Report No. FERMILAB-TM2152, 2001.

[10] L. J. Laslett, in Proceedings of the 1963 summer study on storage rings, Brookhaven National Laboratory, 1963, (BNL, New York, 1963), p. 324; L. J. Laslett and L. Resegotti, in Proceedings of the VIth International Conference on High Energy Accelerators (MIT and Harvard University Cambridge, MA, 1967), p. 150. 
[11] P. J. Bryant, CAS, Report No. CERN-1987-010, 1986.

[12] P. Brunelle, R. Nagaoka, and R. Sreedharan, Measurement and analysis of the impact of transverse incoherent wakefields in a light source storage ring, Phys. Rev. Accel. Beams 19, 044401 (2016).

[13] K. Bane and G. Stupakov, Using surface impedance for calculating wakefields in flat geometry, Phys. Rev. ST Accel. Beams 18, 034401 (2015).

[14] K. Y. Ng, FERMILAB, Report No. FN-0744, 2004.

[15] K. Y. Ng, Physics of Intensity Dependent Beam Instabilities (World Scientific Publishing, Singapore, 2006).

[16] A. Burov and V. Lebedev, Transverse resistive wall impedance for multilayer flat chambers, in Proceedings of the 8th European Particle Accelerator Conference, Paris, 2002 (EPS-IGA and CERN, Geneva, 2002).

[17] N. Mounet, EPFL, Lausanne, Report No. CERN-THESIS2012-055, 2012.
[18] G. Bassi, A. Blednykh, and V. Smaluk, Self-consistent simulations and analysis of the coupled-bunch instability for arbitrary multibunch configurations, Phys. Rev. Accel. Beams 19, 024401 (2016).

[19] Brookhaven National Laboratory, 2006, NSLS-II Conceptual Design Report, https://www.bnl.gov/nsls2/project/ CDR/Cover.pdf.

[20] Handbook of Accelerator Physics, and Engineering, edited by A. W. Chao and M. Tigner (World Scientific Publishing, Singapore, 1999).

[21] A. Blednykh et al., NSLS-II commissioning with $500 \mathrm{MHz}$ 7-Cell PETRA-III cavity, in Proceedings of the 5th International Particle Accelerator Conference, Dresden, 2014.(EPS-AG, Mulhouse, 2014).

[22] A. Blednykh et al., First collective effects measurements in NSLS-II with IDs, in Proceedings of the 6th International Particle Accelerator Conference, Richmond, 2015. (JACoW, Richmond, VA, 2015). 